\title{
Evaluation of the Quality of the Renovation Layers Applied by Thermal Spraying Technology
}

Janette Brezinová(SK) janette.brezinova@tuke.sk

Anna Guzanová(SK) anna.guzanova@tuke.sk

Branislav Hadzima (SK) branislav.hadzima@fstroj.uniza.sk

\section{BIOGRAPHICAL NOTES}

Janette Brezinová, assoc. prof. Ing. PhD. (born in 1968) is currently associate professor and associate dean for education at the Technical University of Košice, Faculty of Mechanical Engineering, Department of Materials and Technology. She graduated in Welding and Surface Treatment (1991), obtained the Ph.D. in the field of degradation in abrasive blast cleaning at the Technical University of Košice in 2003 and in 2008 was habilitated in the field of Mechanical Engineering and Materials. In her research work she focuses on surface treatment and materials, degradation phenomena during abrasive blast cleaning and shot peening, corrosion of metals, corrosion protection of metals, evaluation of organic and inorganic coatings, renovation of worn surfaces. J. Brezinová is the member of Slovak Metal Science Society. She is the author of more than 100 scientific works presented in journals and in proceeding from many international scientific conferences.

Anna Guzanová, Ing. PhD. (born in 1974) is currently assistant professor at the Technical University of Košice, Faculty of Mechanical Engineering, Department of Materials and Technology. She graduated in Welding and Surface Treatment (1997), obtained the Ph.D. in the field of abrasive blast cleaning using ecological types of blasting abrasives at the Technical University of Košice in 2003. In her research work she focuses on surface treatment and materials, shot peening, abrasive blast cleaning, wear of materials and coatings, evaluation of quality the various types of blasting abrasives, evaluation of thermal sprayed coatings. A. Guzanová is the member of Slovak Metal Science Society. She is the author of more than 80 scientific works presented in journals and on many international scientific conferences.

Branislav Hadzima, assoc. prof. Ing. PhD. (born in 1976) is currently a research scientist at the University of Žilina, Faculty of Mechanical Engineering, Department of Materials Engineering. He graduated in Materials science (1999), obtained the Ph.D. in the field of Threshold states of Materials at the University of Žilina in 2003 and in 2009 was habilitated in the field of Materials engineering. In his research work he focuses on corrosion of metals and corrosion protection of metals mainly on corrosion of light metals alloys. B. Hadzima spent his research stays at the University of Technology in Clausthal, Germany and at the Charles University in Prague, Czech Republic. He is the author of the book entitled "Basics of electrochemical corrosion of metals" and more than 110 scientific publications, which were cited more than 160 times. 


\section{KEY WORDS}

BOF - Basic Oxygen Furnace, Thermal Spraying, EIS - Electrochemical Impedance Spectroscopy, Microhardness, Erosive Wear, Mass Loss

\section{ABSTRACT}

This contribution deals with research results focused on analysis of renovative layers applied by thermal spraying. There were two types of sprayed wires used - formerly used S-NiCro 80/20 and new cored wire marked DURMAT. Micro hardness of thermally sprayed coatings was measured by Vickers indenter, wear resistance of coatings was checked by erosion test, simulated by abrasive grit blasting. EDX analysis proved the presence of particular structural phases. Higher micro hardness values were found in DURMAT coating and this property led to higher wear resistance of this coating in comparison with previously used S-NiCro $80 / 20$ coating. Test results showed that new coating marked DURMAT-AS-7641 has better properties in comparison with previously used coating from perspective of chemical composition, micro hardness and also erosive wear evaluation. Part of the experimental work was to determine the electrochemical characteristics of blasted surfaces using the EIS (electrochemical impedance spectroscopy) method.

\section{INTRODUCTION}

Shortage of raw materials in world market and its more difficult and more expensive procuring raises efforts to increase products' lifetime and saving of raw materials and energy. Industrial development is connected mainly with development of material-technical basis with its technology and technical modernization and with substantial advance in labour productivity. Economic reasons of maximal exploitation of materials in mechanical and metallurgical production are actual topics of scientific research nowadays.

The most frequent causes of parts and construction failures are tribology processes occurring on functional surfaces. Tribological characteristics of used materials are of great importance for correct operation of parts and construction nodes. Interaction of functional surfaces during their mutual motion causes undesirable changes on surface layers giving birth to wear. In term of material loss prevention, the most important concern should be active surface protection technology in friction nodes. In steel production, inner parts of oxygen converter hood are subject to extreme wear. Converter hood material is exposed to set of unique factors related to temperature and gas rate variation, dust particles load etc. In addition to extensive heat increase, the material is subject to cyclic load and overheating. With high probability, overheating is the main reason of cracking problem as a result of thermal fatigue caused by thermal cycles from one heat campaign to other, along with differences in converter wall temperature during cooling. Other important degradation factor is erosion caused by oxides of iron, calcium, magnesium, silicium and other elements occurring in exhaust gas. As a result of the inner wall material mass loss by erosion, wall thickness is reduced. For inner hood side protection there are various types of protective coatings used with aim to enlarge hood lifespan. $[1,2,3,4,5,6,7]$.

Suitable surface pretreatment significantly affects the quality of subsequently applied thermal sprayed coatings. Abrasive blast cleaning is appropriate and preferentially used technology in practice for obtaining a clean surface with the desired microgeometry. Blasted surface creates conditions for perfect anchoring of subsequently applied coatings, however, it is characterized by high surface activity. In real conditions, its activity rapidly decreases because of the chemical adsorption of gases from the atmosphere and oxidation. The consequence is an electrode potential reduction of metal and the adhesion of applied coatings. One of the modern methods of corrosion monitoring for determination of electrochemical properties of the surface is electrochemical impedance spectroscopy (EIS) $[8,9,10,11]$. EIS is suitable technique for the measurement of low conducted or complex layers and coatings on the surface of a basic metal. One of the advantages of EIS method is small influence of the measured surface in comparison with voltametric (e.g. potentiodynamic) techniques. EIS resulted in polarization resistance value of the layer on metal surface, which characterizes its corrosion resistance.

This report deals with research results focused on analysis of renovative layers applied by thermal spraying. Part of the experimental work was to determine the electrochemical characteristics of blasted surfaces using the EIS method. 


\section{Materials and Methods}

The specimens for electrochemical measurements have a cylindrical shape $(\varphi 11,3 \times 20 \mathrm{~mm})$ and were manufactured by turning and then they were finished with standard metallographic procedure. The faces of the samples were blasted with mineral blasting material and for comparison also by the glass beads. Pre-treatment was realised on laboratory air blast equipment TVJP - 320 at a pressure of $0,4 \mathrm{MPa}$ until surface saturation.

Electrochemical characteristics of the surfaces were evaluated using EIS on VoltaLab 10 laboratory measuring device. The measurements were performed using PGZ 100 measurement unit and CTV 101 control unit of rotating electrode. Corrosive environment consisted of $0,1 \mathrm{M} \mathrm{NaCl}$ solution. Stabilisation time at the free electrode potential of sample in the electrolyte was 5 min., 1,4 and 8 hours. The frequency varied in the range from 100 $\mathrm{kHz}$ to $10 \mathrm{mHz}$ with change in frequency 20 times per decade. Amplitude of the AC voltage was 20 $\mathrm{mV}$. The temperature during measuring was $22^{\circ} \mathrm{C} \pm$ $1^{\circ} \mathrm{C}$. During the measurement, the sample rotated on the rotating electrode at $70 \mathrm{~min}^{-1}$. Determination and calculation of the polarization resistance Rp was performed from measured EIS courses using circular regression of Nyquist diagrams. For analysis of the measured curves equivalent circuit given in (Fig. 1), was used. The values of parameters that characterize the individual components in the equivalent circuit were determined using VoltaMaster 4 software analysis program. Connection and measurement principle is given in [8].

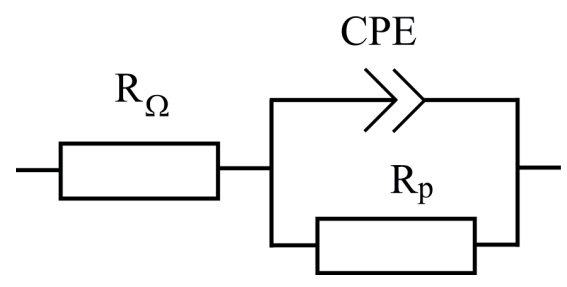

Fig. 1 Equivalent circuit of a simple corrosion system

Surface pre-treated using angular blasting medium is characterised by a lot of sharp notches, its morphology is suitable for coating application, because it allows sufficient anchoring effect. Therefore, for the pretreatment before applying coatings angular blasting medium - brown corundum was selected.
Experimental protective layers were applied on the base material of STN 412021 (ISO 2605) - heat-resistant carbon steel with specific properties, used for making pipes for energy and chemical equipment, operating at high temperatures. [12]

\section{Mechanical properties of base material:}

- Tensile strength: $R m=340-370 \mathrm{MPa}$

- Notch toughness KCU 3:69J. $\mathrm{cm}^{-2}$

- Yield strength at $20^{\circ} \mathrm{C}: 235 \mathrm{MPa}$

- Brinell hardness: max. 147HB

- Elongation A5: $\min .25 \%$

- Modulus of elasticity $\mathrm{E}$ at $20^{\circ} \mathrm{C}$ : $\mathrm{min} .206 \mathrm{GPa}$

Chemical composition of base material is shown in (Tab. 1).

\begin{tabular}{|c|c|c|c|}
\hline $\mathbf{C}$ & $\mathbf{M n}$ & $\mathbf{S i}$ & $\mathbf{C r}$ \\
\hline $0,07-0,15$ & $0,35-0,60$ & $0,77-0,35$ & $\max .0,25$ \\
\hline $\mathbf{N i}$ & $\mathbf{C u}$ & $\mathbf{P}$ & $\mathbf{S}$ \\
\hline $\max 0,25$ & max. 0,25 & max. 0,04 & max. 0,04 \\
\hline
\end{tabular}

Tab. 1 Chemical composition of base material (wt-\%)

There were two types of cladding layers applied by thermal spraying technology on this base material. Characteristics of applied materials:

1. S - NiCro 80/20 in form of $\varnothing 1,6 \mathrm{~mm}$ wire, manufactured by Zander Schweisstechnik, applied by thermal flame-spraying technology. Chemical composition is shown in (Tab. 2). This cladding material was applied in two layers.

\begin{tabular}{|c|c|c|c|c|}
\hline $\mathbf{C}$ & Si & Mn & S & Cr \\
\hline 0,036 & 1,19 & 0,29 & 0,002 & 19,8 \\
\hline
\end{tabular}

Tab. 2 Chemical composition of S - NiCro 80/20 (wt-\%)

2. DURMAT - AS - 761 in form of cored wire $\varnothing 1,6 \mathrm{~mm}$, manufactured by DURUM Verschleißschutz $\mathrm{GmbH}$, applied by wire arc spraying technology. Chemical composition is shown in (Tab. 3).

\begin{tabular}{|c|c|c|c|c|c|}
\hline $\mathbf{C}$ & $\mathbf{S i}$ & $\mathbf{C r}$ & $\mathbf{N i}$ & $\mathbf{B}$ & WSC \\
\hline 0,35 & 4,10 & 10,60 & 33,15 & 1,80 & 50 \\
\hline
\end{tabular}

Tab. 3 Chemical composition of DURMAT - AS - 761 (wt-\%)

Before coatings application, test samples were pretreated by pneumatic abrasive blast cleaning: blasting medium - brown corundum, grain size $0,71 \mathrm{~mm}$, blasting angle $75^{\circ}$, air pressure $0,6 \mathrm{MPa}$, distance 
testing sample - $250 \mathrm{~mm}$ blasting nozzle.

The surface roughness of blasted samples was evaluated by contact roughness tester Surftest SJ - 301, Mitutoyo, according to STN EN ISO 4287 - Profile method. Following roughness parameters were evaluated in mean line measuring system:

$\square a$ (arithmetical mean deviation of the profile), - Rz (maximum height of profile),

- $R q$ (root mean square deviation),

- Rt (total height of profile),

- $R S m$ (mean width of the profile elements).

Roughness measuring parameters were chosen as follows:

asampling length I $(\lambda c)=2,5 \mathrm{~mm}$,

number of sampling lengths $N=5$,

measured profile: $R$ (mean line measuring system),

- filter used: Gauss,

nevaluation length $\mathrm{ln}=12,5 \mathrm{~mm}$.

Vickers microhardness test of cladding and also base material was evaluated on metallographic crosssections according to STN EN ISO 6507, testing equipment DUH 202 SHIMADZU, (Fig. 2).

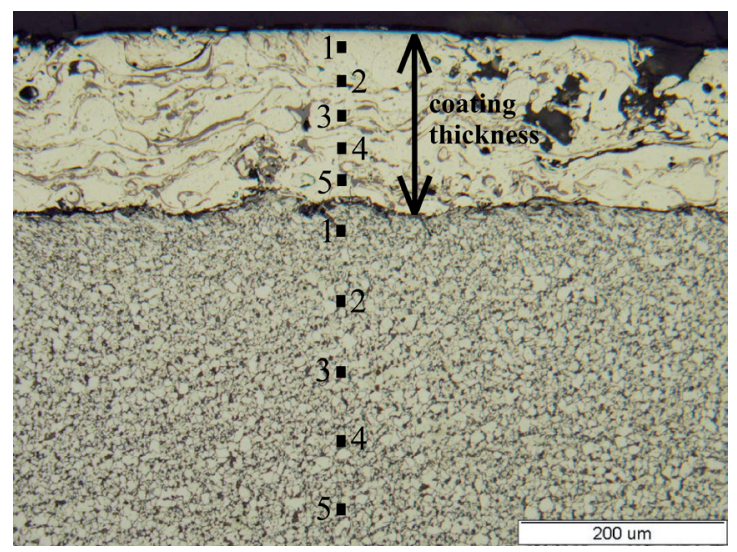

Fig. 2 Pattern of microhardness measurement

Microstructure of evaluated coatings was observed by SEM FEI Quanta 400. Chemical composition of applied coatings was analysed by EDX analysis. Tested coating was exposed to erosive wear simulated by abrasive grit blasting with aim to simulate operating conditions. The equipment used for this aim was laboratory blasting equipment KP-1, which enables to control number of blasting cycles. After each blasting cycle, weight of tested samples was evaluated. Mass loss of tested samples was monitored at two blasting angles: $45^{\circ}$ and $75^{\circ}$. Used blasting medium - brown corundum with grain size
$0,9 \mathrm{~mm}$. Circumferential velocity of blasting wheel $51,0 \mathrm{~m} \cdot \mathrm{s}^{-1}$, output velocity of abrasive grains $-70,98$ $\mathrm{m} . \mathrm{s}^{-1}$. One erosive cycle equals to 5 cycles of blasting medium in blasting equipment.

\section{Results}

Surface of base material after grit blasting with angular blasting medium is characterized by typical morphology - undirected, isotropy surface. This morphology is suitable for functional coating application, because it provides higher adhesion to substrate. Blasted surface roughness parameters are shown in (Tab. 4).

Figure 3 shows appearance of the surface blasted by brown corundum. Impact of large number of blasting grains gives rise to very rugged surface. Its nature depends on several factors. The main factors influencing the nature of the blasted surface are the shape and size of blasting grains, surface coverage degree, parameters of blasting machine and material characteristics of the basic material and the blasting material used.

\begin{tabular}{|l|l|l|l|}
\hline R-profile & \multicolumn{3}{|l|}{} \\
\hline$\lambda m c(m m)$ & 2,5 & $\operatorname{Rq}(n m)$ & 14,3 \\
\hline$N$ & 5 & $\operatorname{Rt}(n m)$ & 93,1 \\
\hline $\operatorname{Ra}(n m)$ & 11,42 & $\operatorname{RSm}(10 \%)$ & 392 \\
\hline $\operatorname{Rz}(n m)$ & 72,89 & & \\
\hline
\end{tabular}

Tab. 4 Surface roughness parameters of blasted surface

Blasted surface belongs to the undirected, isotropic surfaces, which means that the value of surface roughness in the longitudinal and perpendicular direction is not significantly different. The blasted surface is characterized by sharp deep hacks. It is mainly used to obtain the desired surface roughness in the pre-treatment and surface cleaning. From (Fig. 3) it is clear that the surface was completely covered with the notches after blasting grain impact, i.e. the degree of surface coverage equals 1 . The figure also shows remains of blasting grains wedged in the surface.

Nyquist diagrams of steel surface after blasting by glass beads and corundum are reported in (Fig. 4). Polarisation resistance in this form of the measured curves equals to the diameter of the semicircle in Nyquist plot. There was the presence of only one layer recorded. 


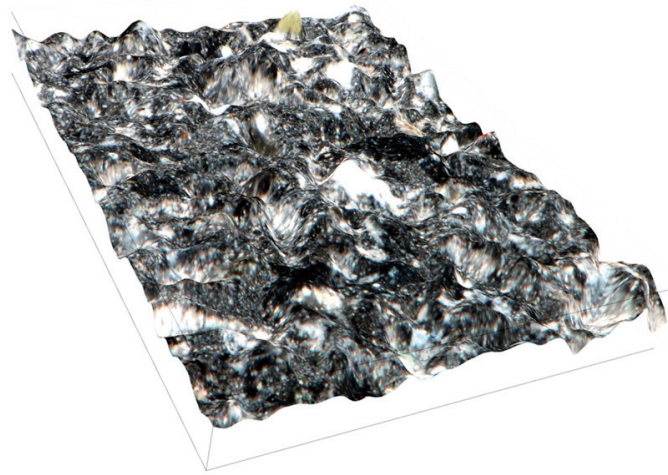

Fig. 3 3D view on surface blasted by corrundum

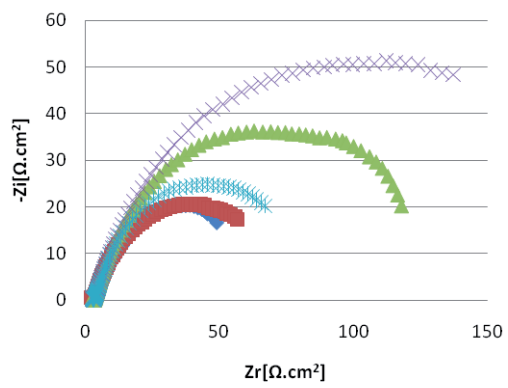

- S235JRG1 5 min

- $5235 J$ RRG1 1 hod.

$\triangle$ S235JRG1 2 hod

$\times$ S235JRG14 hod

* S235JRG1 8 hod

a)

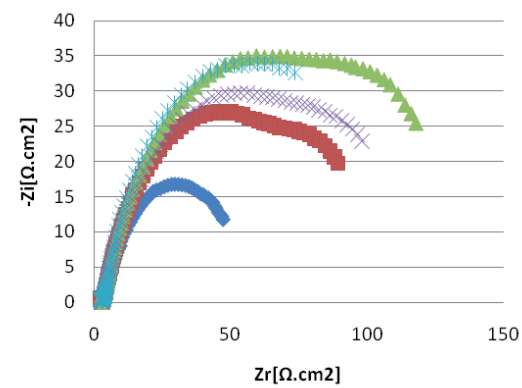

- S235JRG1 5 min

- S235JRG1 1 hod.

$\triangle$ S235JRG1 2 hod

$\times$ S235JRG1 4 hod

* S235JRG1 8 hod.

b)

Fig. 4 EIS curves of S235JRG1 steel in $0.1 \mathrm{M} \mathrm{NaCl}$ solution after blasting by a - glass beads, $b$ - corundum

The value of polarization resistance $\mathrm{Rp}$ increased with the exposure time of samples in the electrolyte, (Fig. 5). Higher values of Rp were measured after application of glass beads, which may be related to the size of the assessed blasted area, but especially to the lower surface roughness of the surface blasted by glass beads and also to lower energy transmitted by impinging particles of glass beads compared to angular brown corundum grit. The maximum value of Rp was recorded for both blasting media after 4 hour exposure of samples in corrosive environment. With the increasing value of Rp corrosion rate decreases. Decrease in polarization resistance after 4 hours of exposure is due to release of corrosion products, which were partially protective in nature, causing the formation of new surfaces and the further development of active corrosion process. Corrosive products are better able to adhere to the blasted surface, and if disrupted faster corrosion processes and the creation of new partially protective corrosion products on the exposed surface occur. The increase in total resistance at the interface of material - electrolyte is due to the increase in resistance of the porous layer.

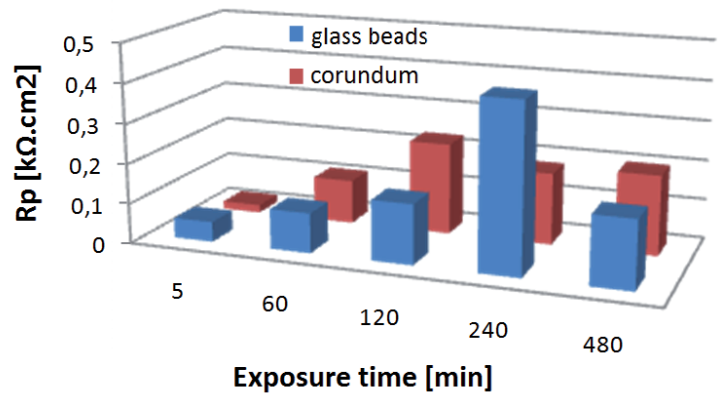

Fig. 5 Diagrams of polarization resistance for the surfaces blasted by different types of blasting media

Experimentally determined microhardness of base material was $281 \mathrm{HV}$ 0.01. Figure 7 shows ferrite - pearlite structure of base material. Microhardness of coating made of DURMAT-AS-761 was higher (810 HV 0.5) in comparison with S - NiCro 80/20 coating (775 HV 0.5), resulting from material properties, chemical composition and structure of particular materials, (Fig. 6). It is possible to assume that higher microhardness values provide higher wear resistance of coatings.

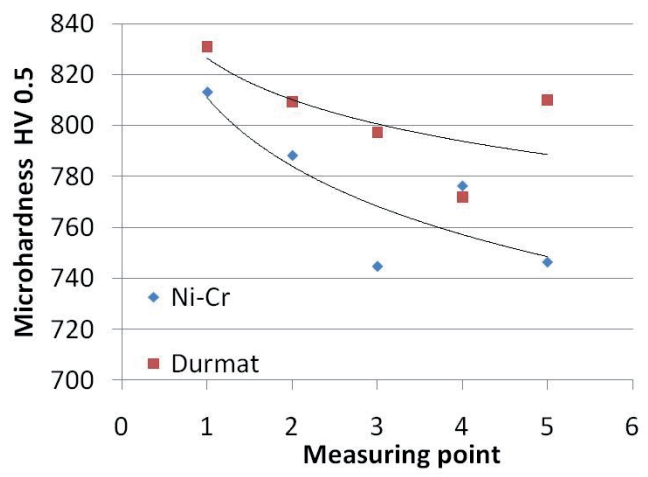

Fig. 6 Microhardness of evaluated coating 


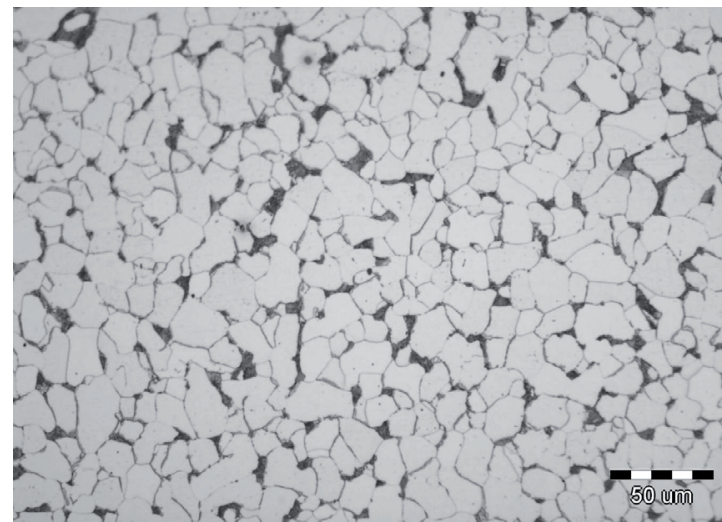

Fig. 7 Structure of base material

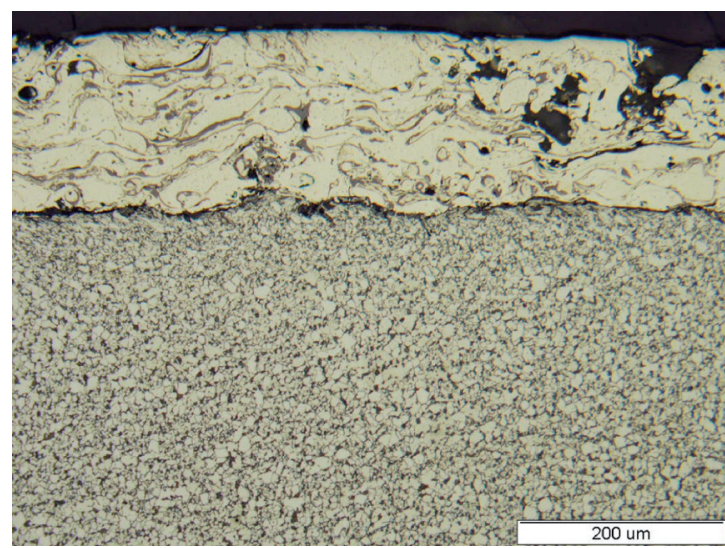

Fig. 8 Cross-section of coating S - NiCro 80/20

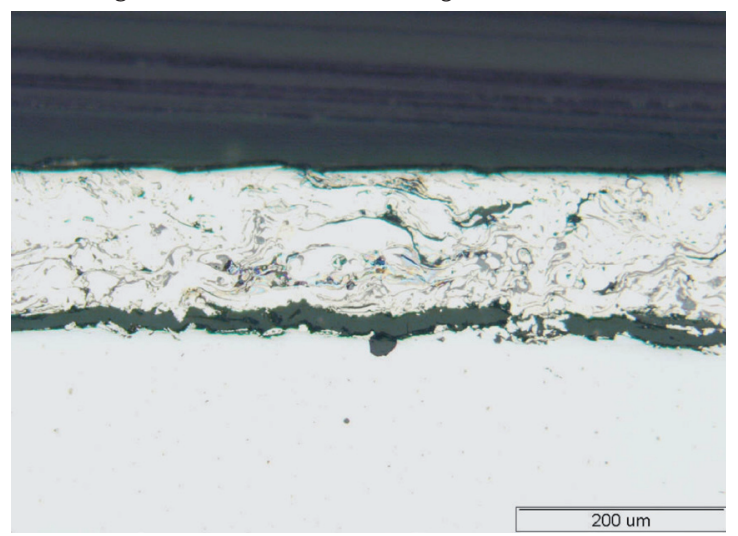

Fig. 9 Cross-section of coating DURMAT- AS - 761

Microscopic analysis proved characteristic sandwich structure of both evaluated coatings, (Fig. 8, 9). Chemical composition of present phases and interlayers was determined by EDX analysis, (Fig. 13). It is possible to observe impinged particle deformation and porosity of coatings. Figures 10 and 11 show interlayer under-coatings formed by $\mathrm{Ni}, \mathrm{Fe}, \mathrm{Cr}$.

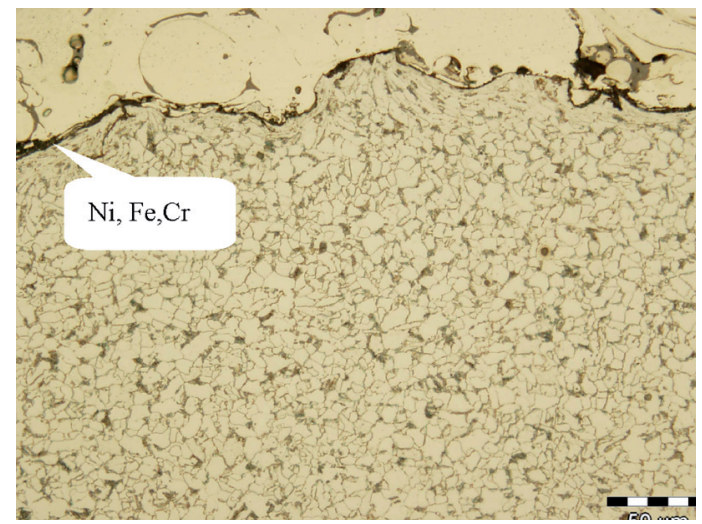

Fig. 10 Interlayer under-coating S - NiCro 80/20

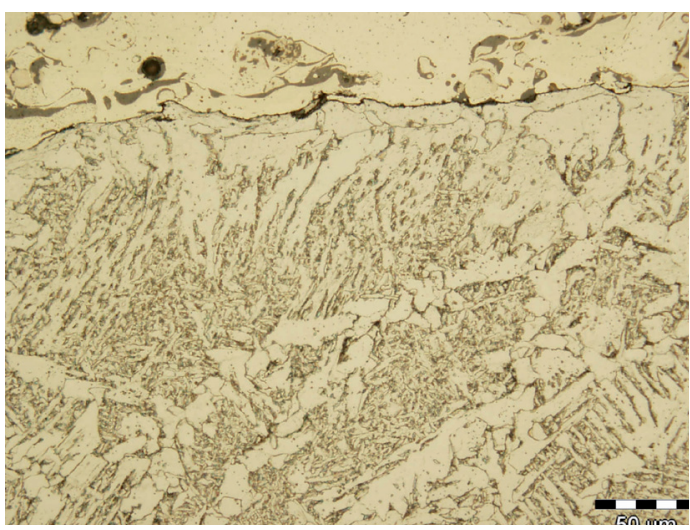

Fig. 11 Interlayer under-coating DURMAT- AS - 761

Utilization of tubular cored wires seems to be suitable for protective coatings, in cases when corrosion and wear occur together. Characteristic feature of the wires is higher melting power and consequently higher coating making productivity. Melting rate of tubular wire depends on current density and wire unwinding rate.

Figure $12 \mathrm{a}$ shows mass loss Wh (g) of samples coated by DURMAT-AS-761 at blasting angle $75^{\circ}$ and $45^{\circ}$, (Fig.12b) shows wear process of $\mathrm{S}$ - NiCro $80 / 20$ coating at mentioned blasting angles. Lower wear of both coating was achieved at $75^{\circ}$ blasting angle. Lower blasting angle $=$ more intensive material wear. Notched abrasive grain can cause crack generation and failure of coatings, resulting in stress concentration and coating separation. Erosive wear test was carried out up to 5 cycles, when coating was completely removed. DURMAT-AS-761 coating showed high wear resistance resulting from its material properties, chemical composition and material structure along with its microhardness. 

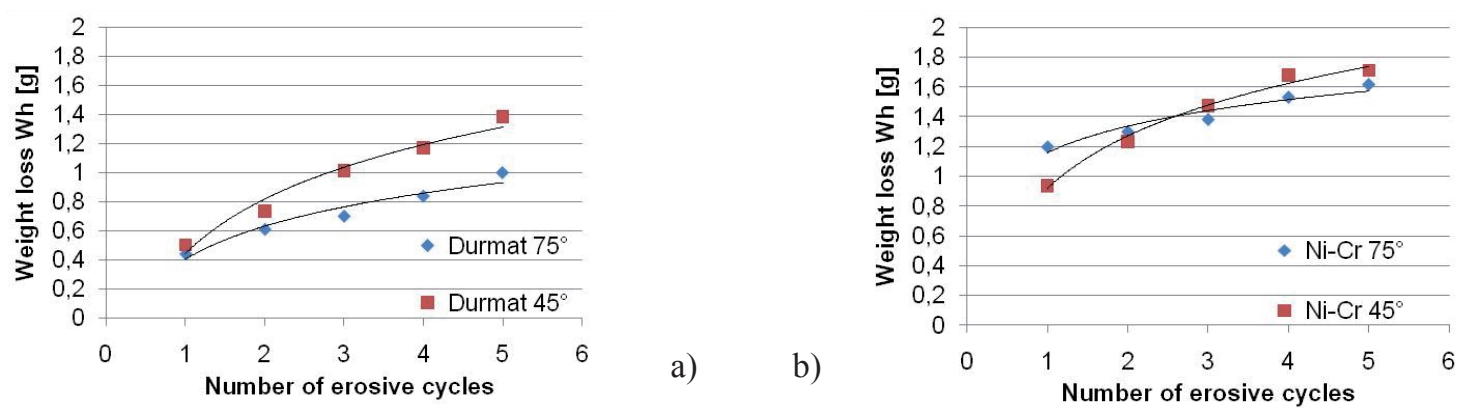

Fig. 12 Mass loss of samples coated by a) DURMAT-AS-761, b) S - NiCro 80/20
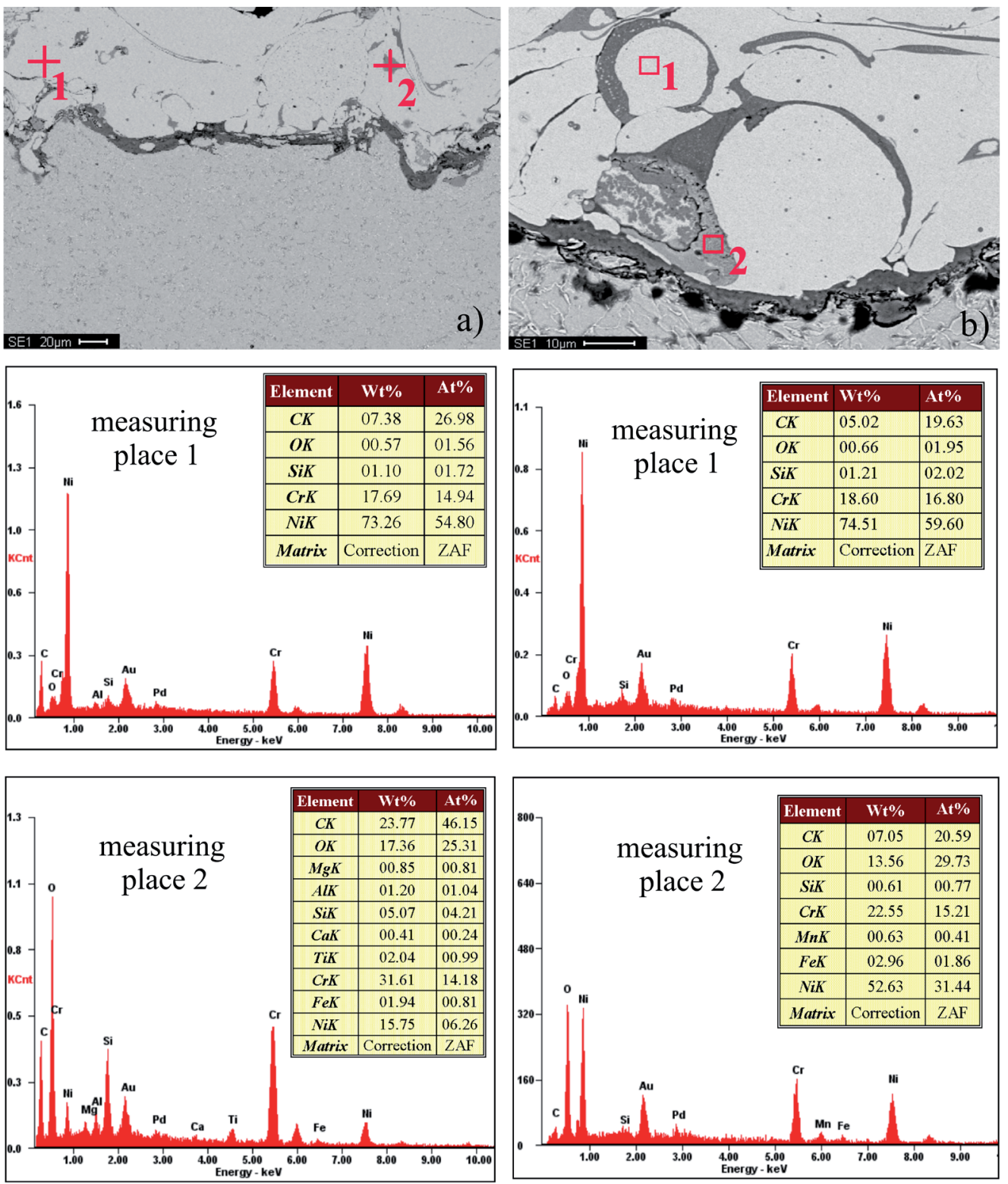

Fig. 13 EDX analysis of coating a) S - NiCro 80/20, b) DURMAT- AS - 761 


\section{Conclusion}

Analysis of Nyquist diagrams and the measured polarization resistance show that the higher value of Rp was present in material blasted by glass beads, which corresponds to a lower corrosion rate. This is in line with the obtained surface morphology. The surface created after blasting with glass beads is composed of spherical indentations after the impact of beads and partly of notches created by fragments of blasting particles. Decrease in polarisation resistance is due to the releasing of corrosion products, which are protective in nature. The rate of corrosion reactions has practical significance in terms of temporary protection of surfaces pre-treated by abrasive blast cleaning until the application of protective and functional coatings.

Experimental work has proved that the use of EIS as a modern non-destructive method presents new opportunities of quick way how to quantitatively describe the local corrosion process, course of corrosion processes and their controlling. It represents the future in industrial monitoring of corrosion.

Problems related to actual need for increasing the lifetime of oxygen converter hood gave rise to this report. During steel making, there is combined wear of cooling hood pipes occurring. Exposed parts of hood are exploited by high-temperature corrosion along with erosive and abrasive wear by flowing particles consisting of iron, oxides of iron etc. Thermally sprayed coatings are applied to increase lifetime of cooling pipes and to protect the base material. The aim of this report was to analyse currently used S - NiCro 80/20 coating and compare it with new DURMAT-AS-761 coating. Tested samples were cut out of functional hood parts.

Microscopic analysis proved typical sandwich structure on both of the evaluated coatings. EDX analysis determined chemical composition of present phases and interlayers.

Experimentally determined microhardness of the base material was $281 \mathrm{HV}$ 0.01. Microhardness of new tested coating, DURMAT-AS-761, was higher (810 HV 0.5), in comparison with S - NiCro 80/20 coatings ( $775 \mathrm{HV} \mathrm{0.5);} \mathrm{and} \mathrm{it} \mathrm{results} \mathrm{from} \mathrm{their} \mathrm{ma-}$ terial characteristics, chemical composition and structure. It can be assumed that higher microhardness values provide higher wear resistance of coatings.

Erosive wear test proved lower mass loss at $75^{\circ}$ blasting angle for both of evaluated coatings.
Lower blasting angle causes more intensive material reduction. At places of abrasive impact, some cracks and coating failures can appear resulting in stress concentration and coating separation. Achieved results showed, that new coating marked DURMAT-AS-761 has better properties in comparison with previously used coating from perspective of chemical composition, microhardness and also erosive wear evaluation.

\section{Acknowledgement}

This contribution is the result of the project implementation: „Unique equipment for evaluation of tribocorrosion properties of the mechanical parts surfaces" (ITMS: 26220220048) supported by the Research \& Development Operational Programme funded by the ERDF and project No.1/0510/10 supported by grant agency VEGA.

\section{References}

[1] Rigney, D. W., Viguie, R., Wortman, D. J., Skelly, D. V.: Thermal barrier coatings applications and process development for aircraft engines. Journal of thermal spray technology 6, 1997, pp. 167-175

[2] Diekmann, H., Gramberg U.: The Role of Tantalum as a Construction Material in the Chemical Industry. Annual Symposium of the Tantalum International Study Center, Wien, Oct. 4 - 6, 1994

[3] Vasen, R., Pracht, G., Stover, D.: New thermal barrier coating systems with a graded ceramic coating. ITSC 2002, Essen 2002

[4] Pershin, V., Lufitha, M., Mostaghimi, J., Chandra, S.: Effect of substrate temperature on nickel coating adhesion. Proceedings from Materials solutions 2001, Thermal spray symposium, Ottawa 2001

[5] Cherico, S., Toth, R.: Survey of various protective coatings and deposition technology to exhaust cover BOF lifespan extension. Cleveland, Ohio, 2006

[6] Ambrož, O., Kašpar, J.: Plasma sprayed coatings and their utilization in industry. Brno, 1990, $320 \mathrm{~s}$

[7] Amada, S., Hirose, T.: Influence of grit blasting pre-treatment on the adhesion strength of plasma sprayed coatings: fractal analysis of roughness. Surface and Coatings Technology, 102,1998, s.132-137 
[8] Škublová, L. - Mráziková, R. - Škorík, V.: Vplyv elektroerozívne upraveného povrchu na koróznu odolnost' titánovej zliatiny Ti6Al4V. Transfer inovácií, č.17/2010, 2010, pp. 116-119

[9] Nauer, G. E.: Modern electrochemical surface treatments for automotive applications, Kplus ECHEM, University of Viena, 2008

[10] Raja, V.S. et al.: Electrochemical impedance behavior of graphite-dispersed electrically conducting acrylic coating on AZ31 magnesium alloy in 3.5 wt.\% NaCl solution. Progress in Organic Coatings, Volume 67, Issue 1, January 2010, Pages 12-19

[11] http://www.gamry.com/App_Notes/EIS_ Primer/EIS_Primer.htm\#Model2

[12] Egri, M.: Application of specific surface treatments with aim of extension of mechanical parts lifespan. Diploma thesis. Technical university of Košice, 2008

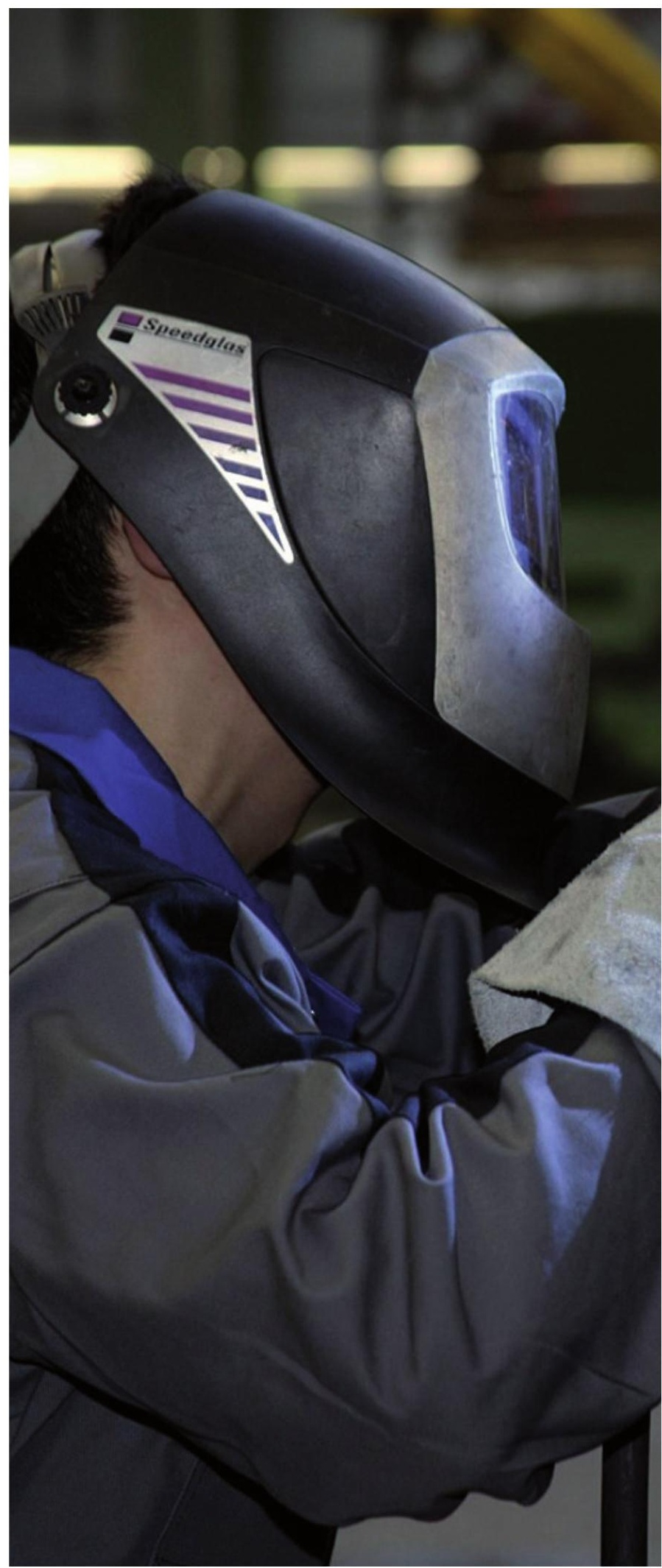




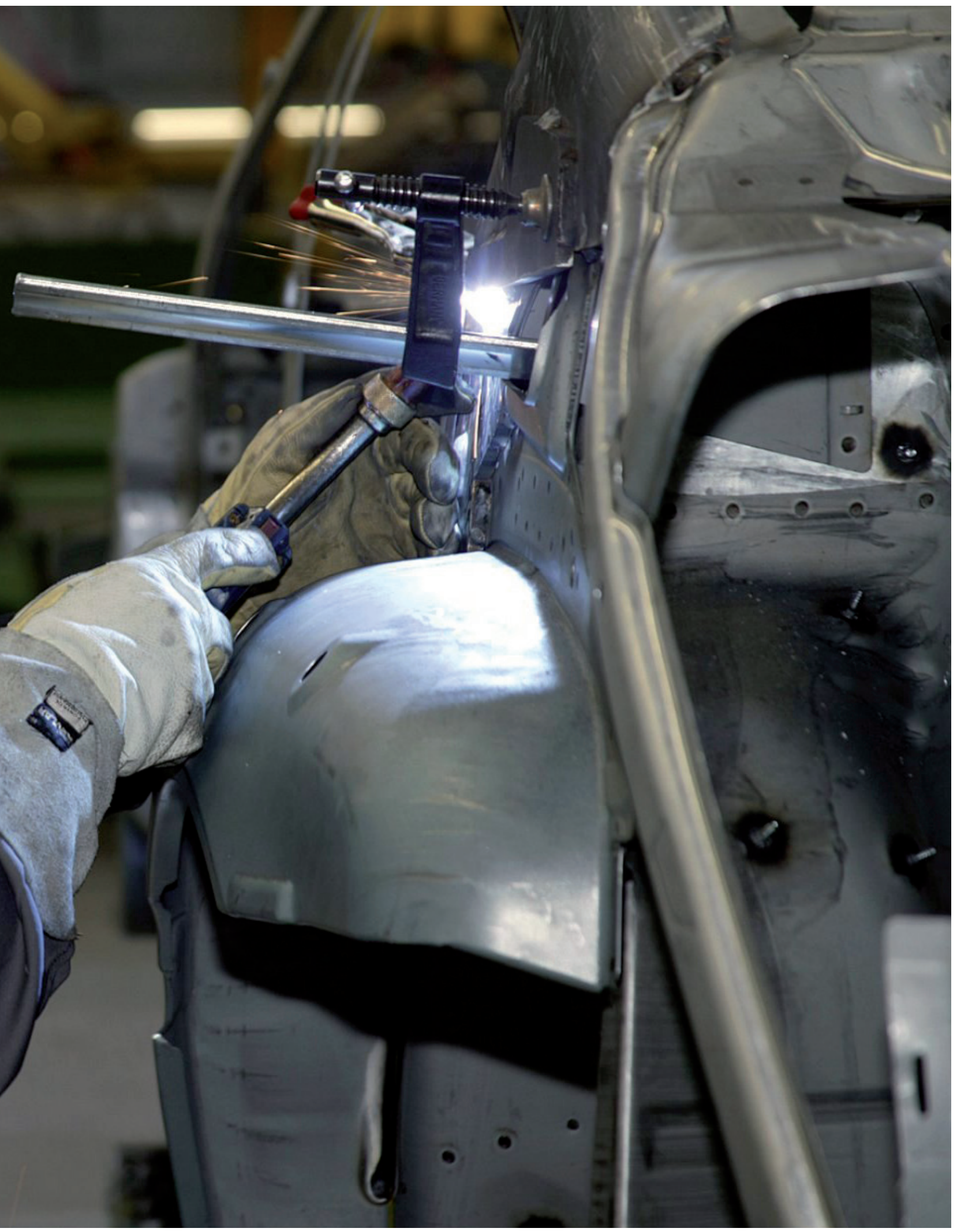

